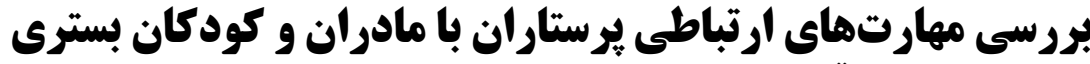

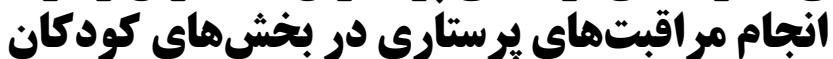

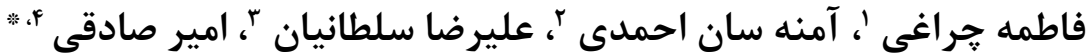

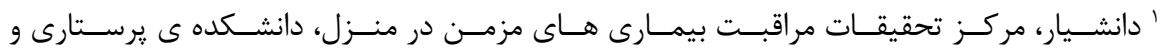

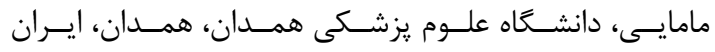

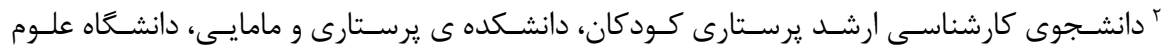

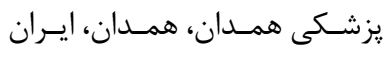

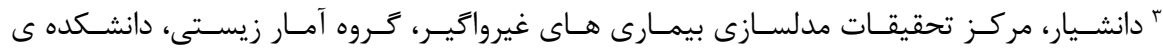

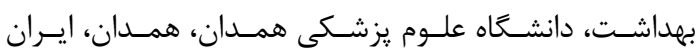

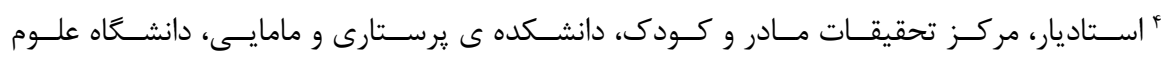

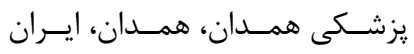

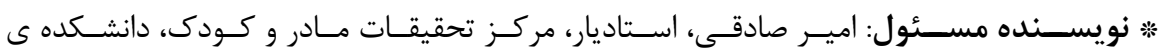

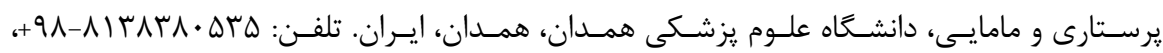

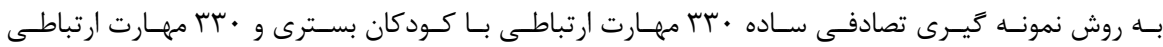

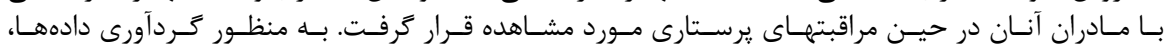

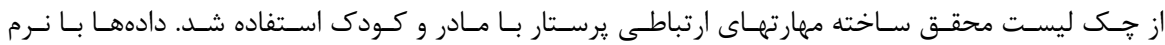

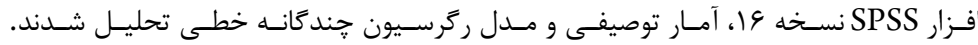

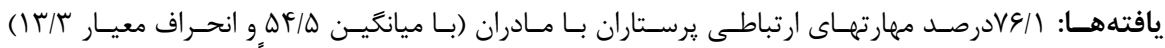

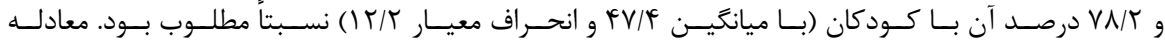

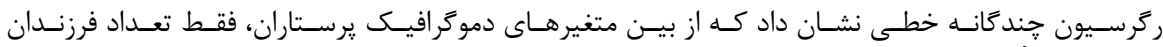

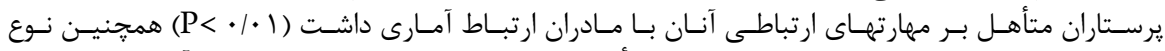

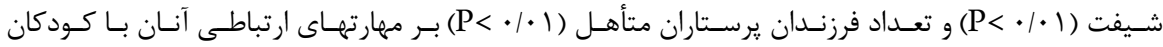

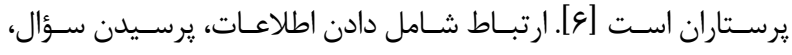

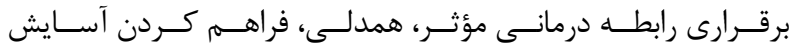

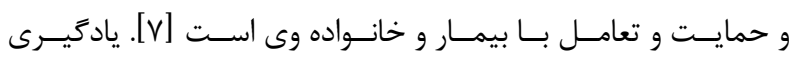

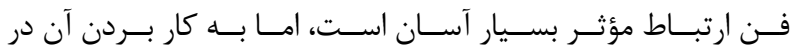

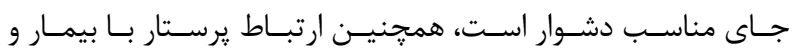

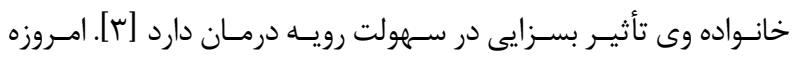

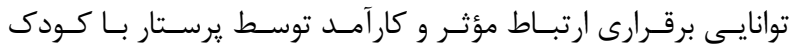

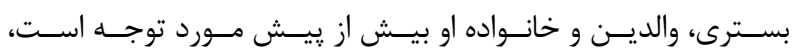

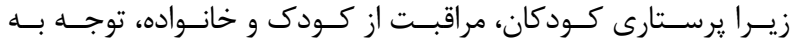

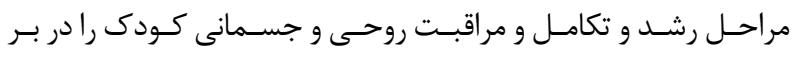

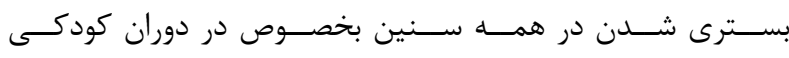

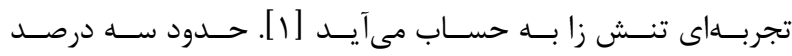

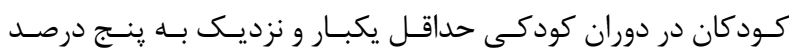

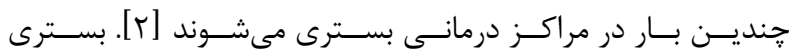

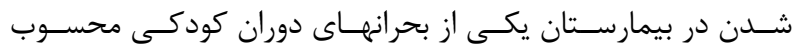

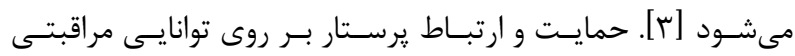

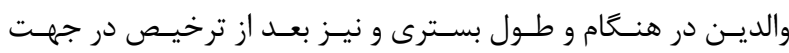

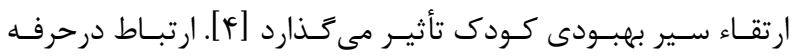

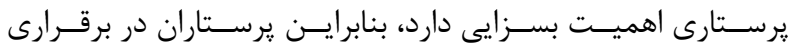

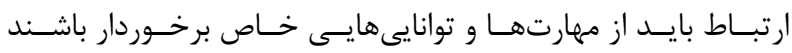


يرسـتاران بـا ارتبـاط نزديـك بـا مـادر كـودى بسـترى مى تواننـد

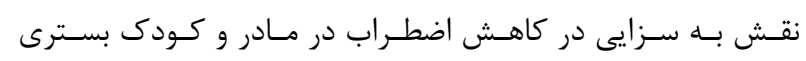

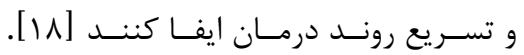

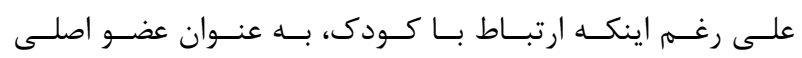

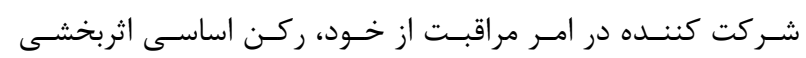

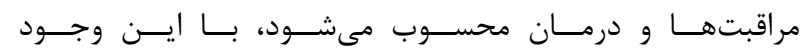

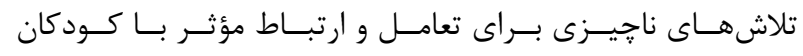

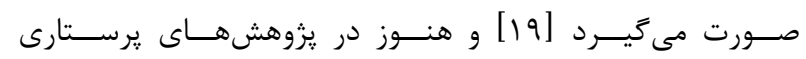

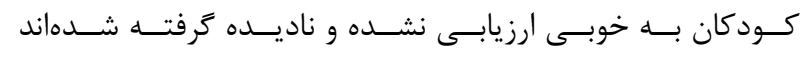

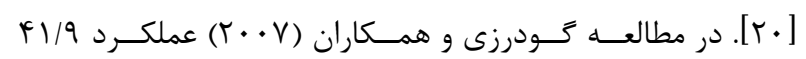

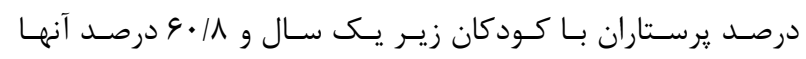

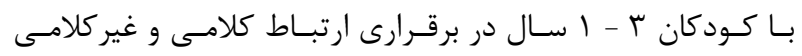

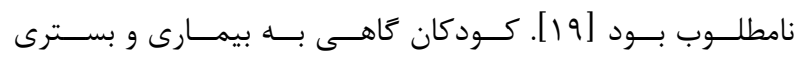

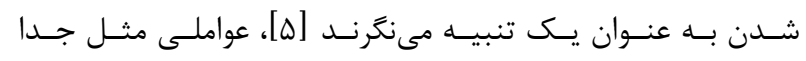

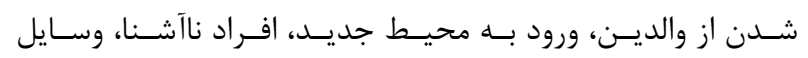

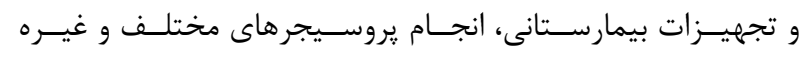

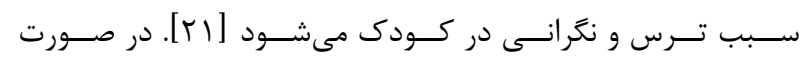

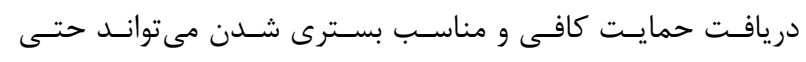

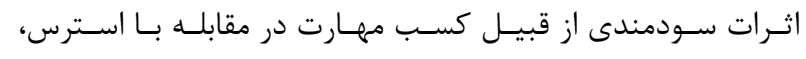

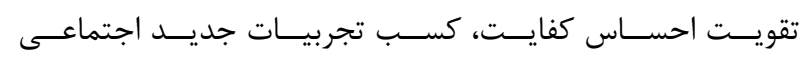

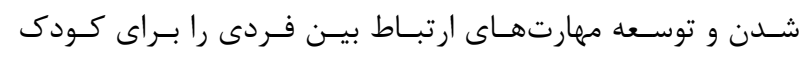

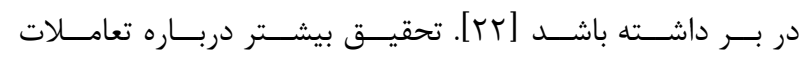

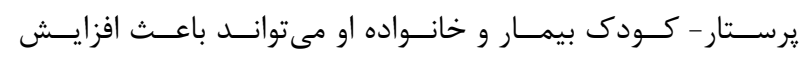

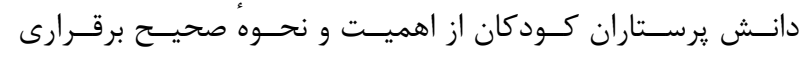

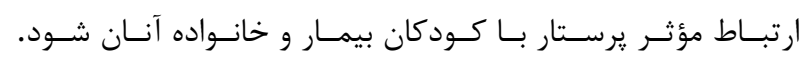

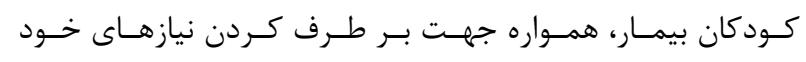

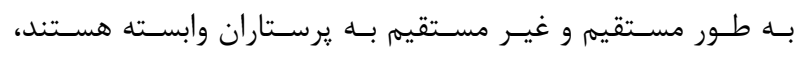

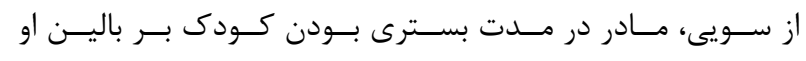

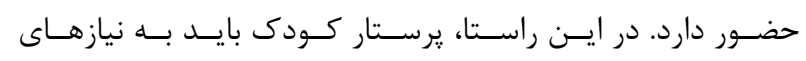

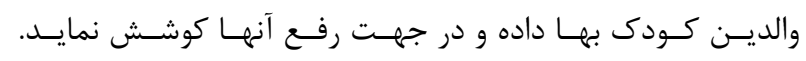

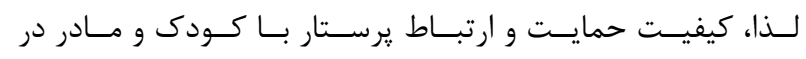

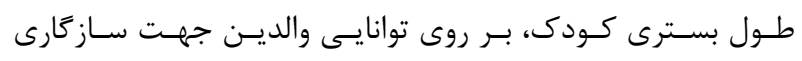

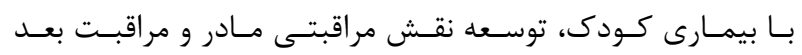

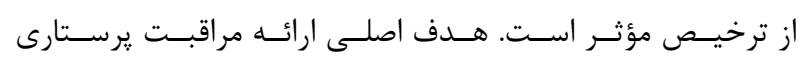

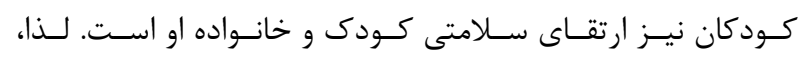

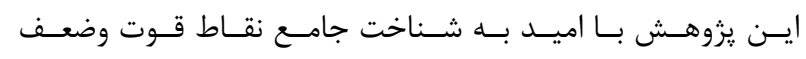

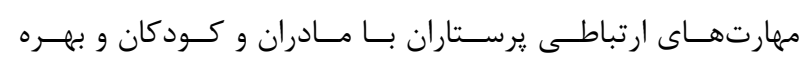

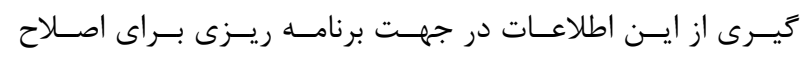

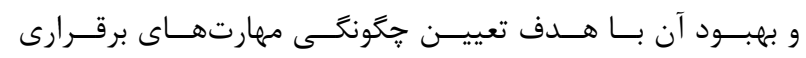

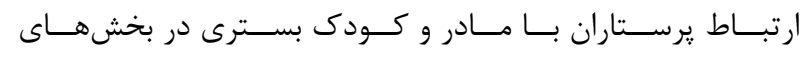

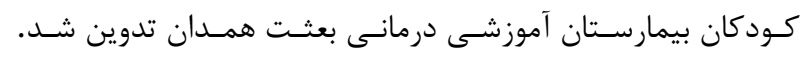

مى گيـرد [N]. لـذا، عـلاوه بــر رفـع نيازهـاى كـودكان بيمـار، يرسـتار

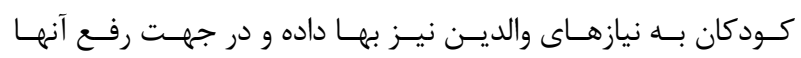

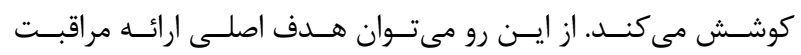

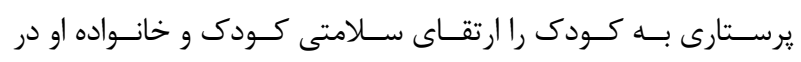

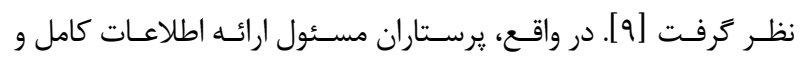

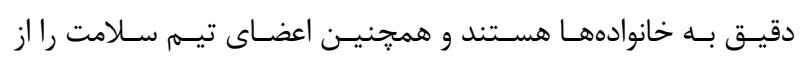

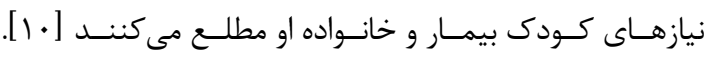

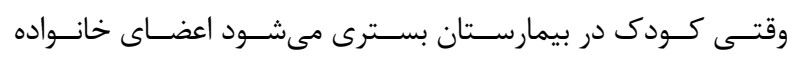

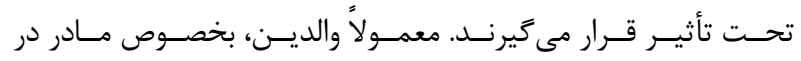

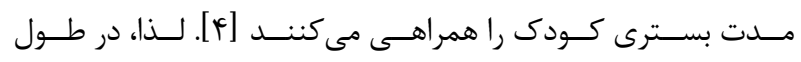

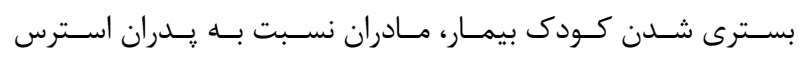

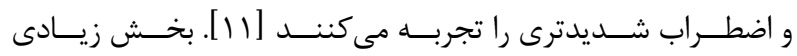

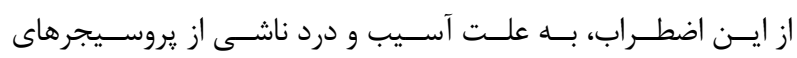

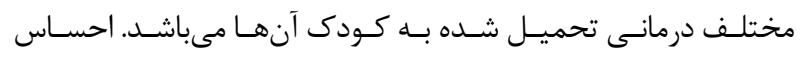

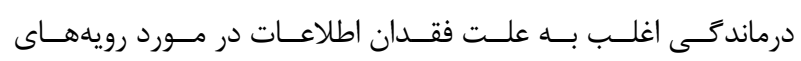

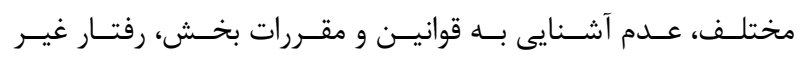

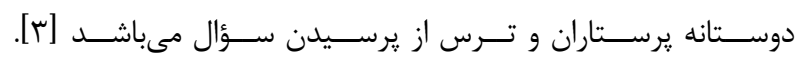

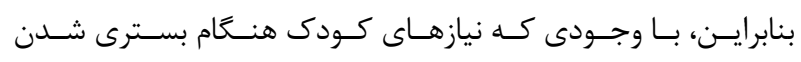

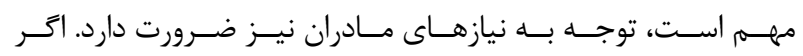

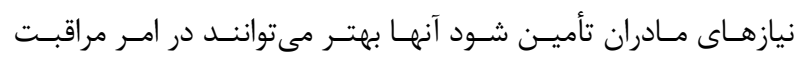

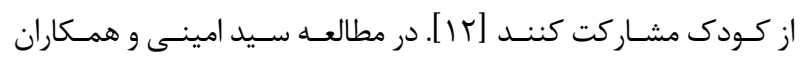

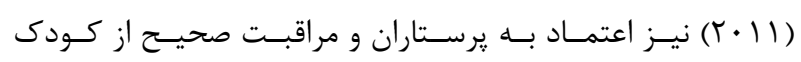

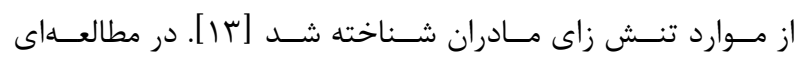

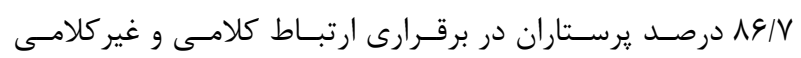

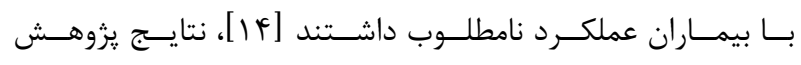

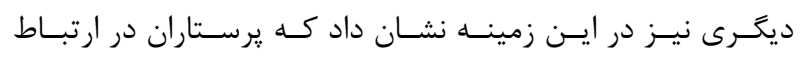

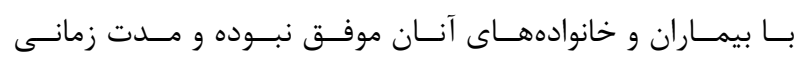

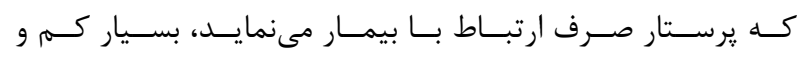

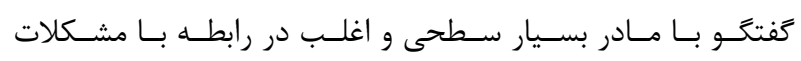

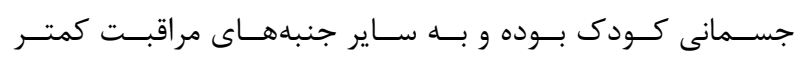

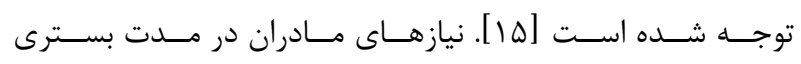

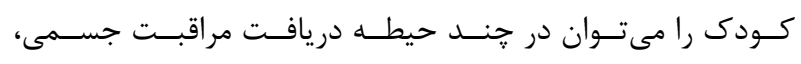

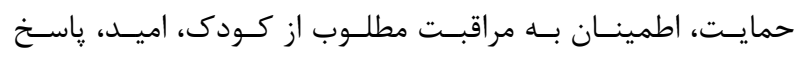

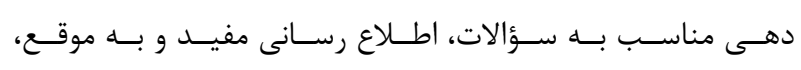

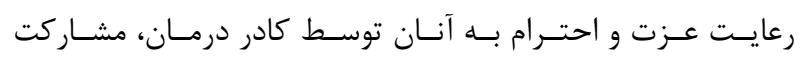

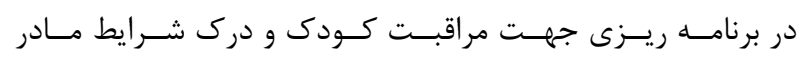

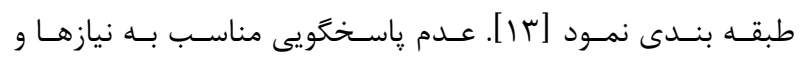

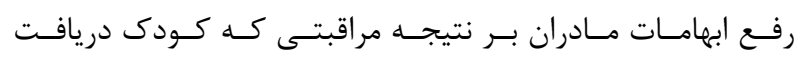

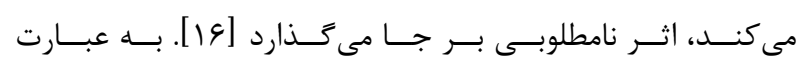

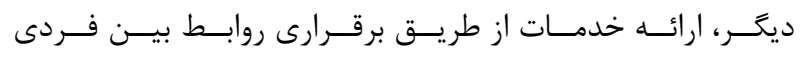

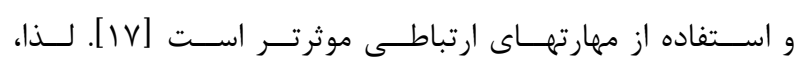




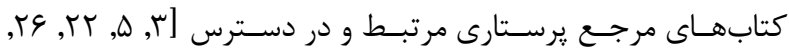

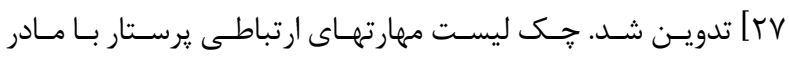

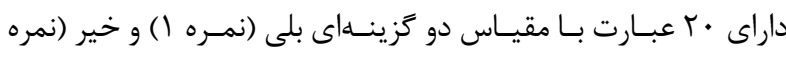

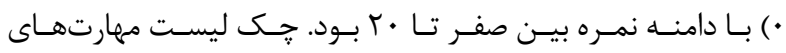

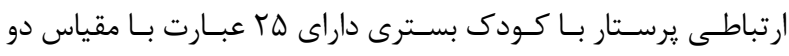

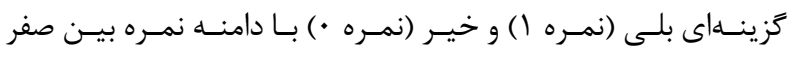

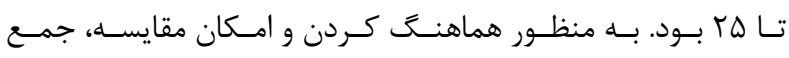

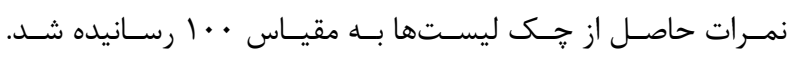

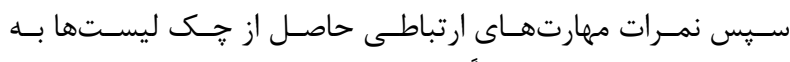

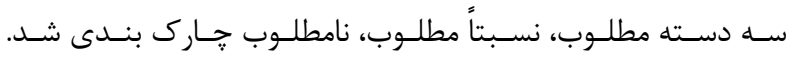

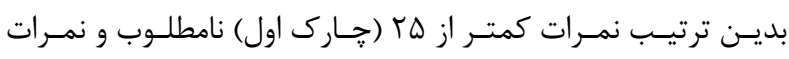

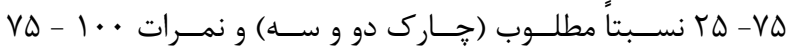

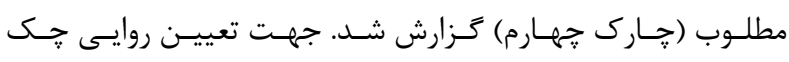

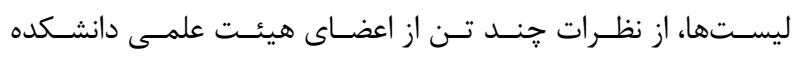

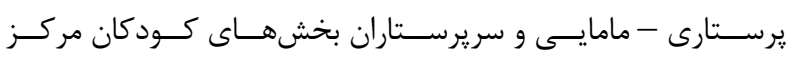

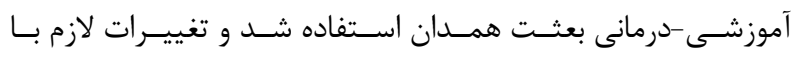

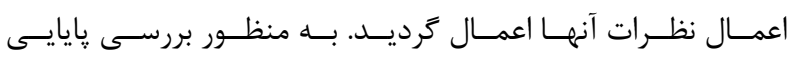

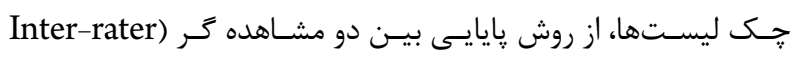
(reliability

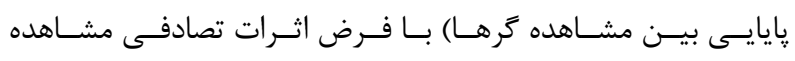

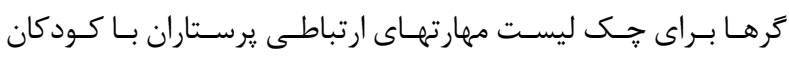

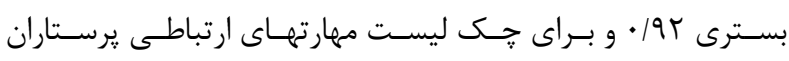

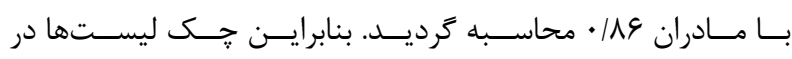

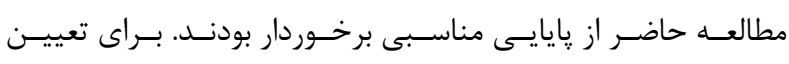

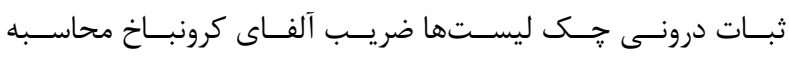

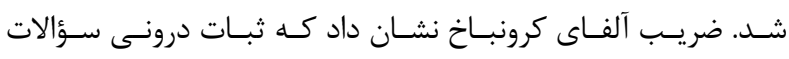

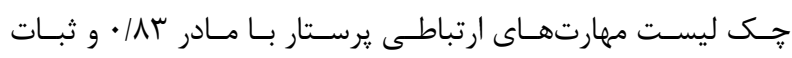

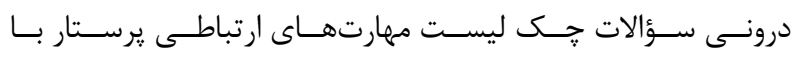

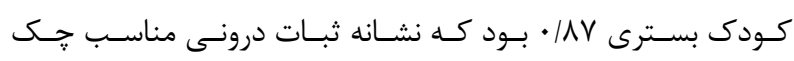

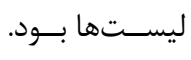

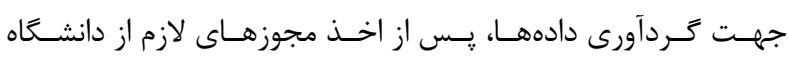

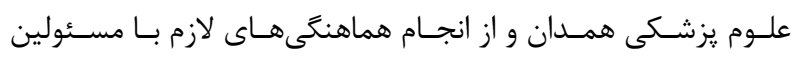

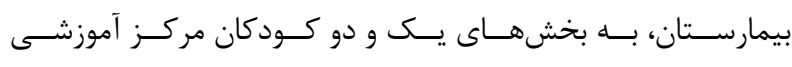

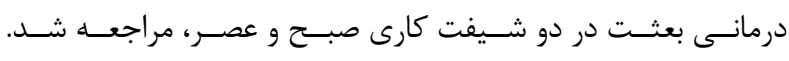

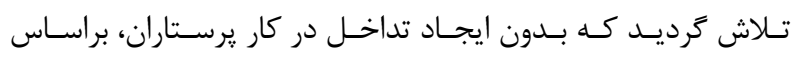

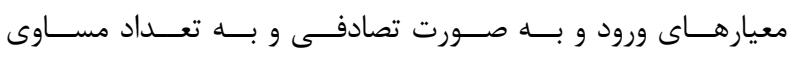

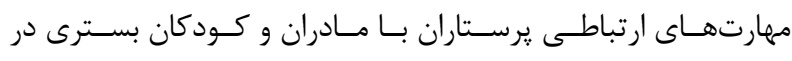

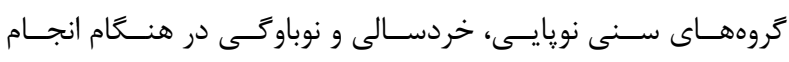

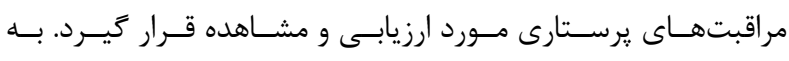

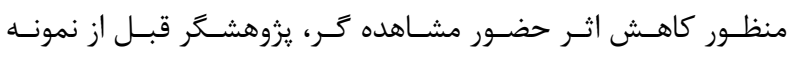

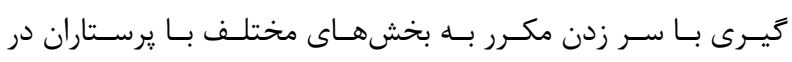

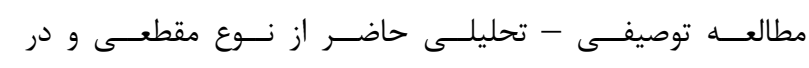

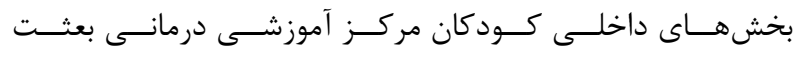

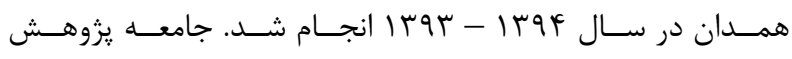

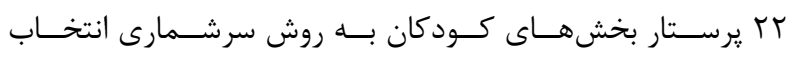

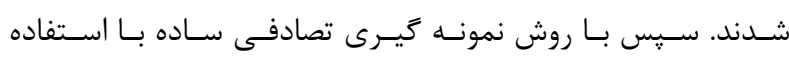

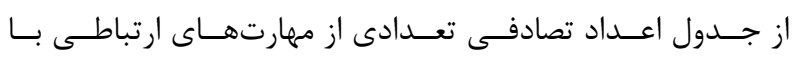

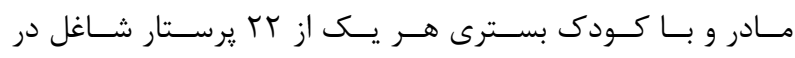

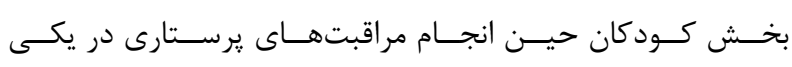

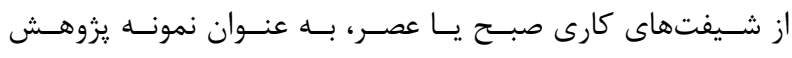

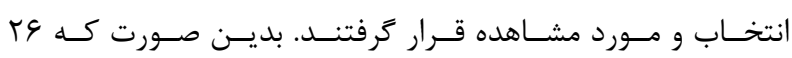

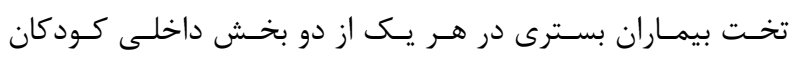

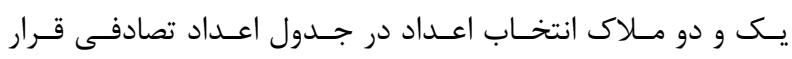

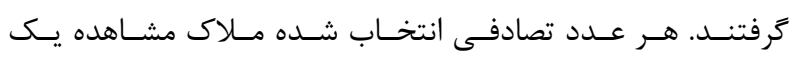

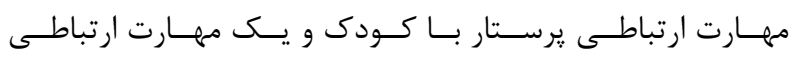

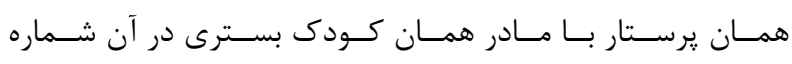

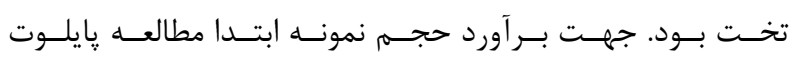

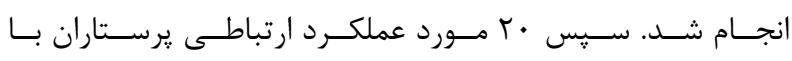

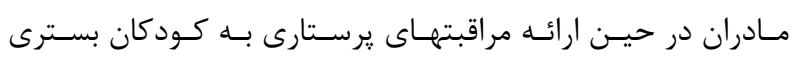

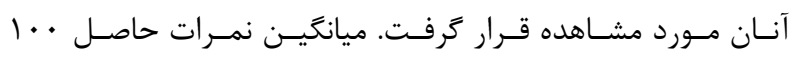

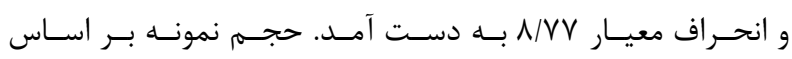

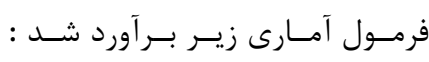

$$
\begin{aligned}
& \mathrm{n}=Z^{r} 1-\alpha / r(\delta)^{r} / \alpha^{r}
\end{aligned}
$$

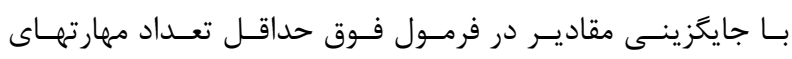

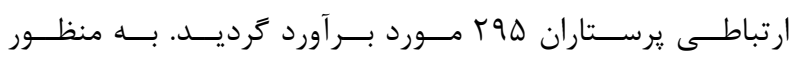

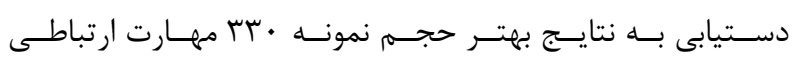

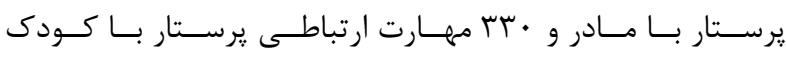

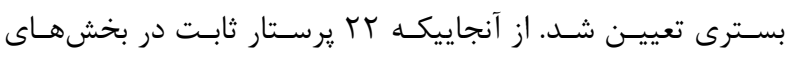

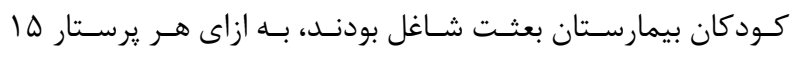

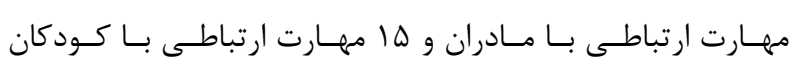

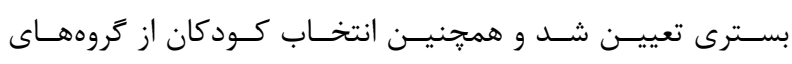

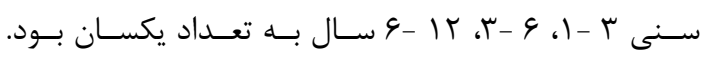

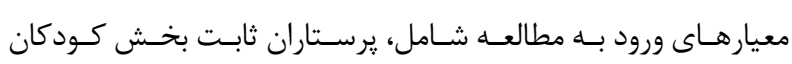

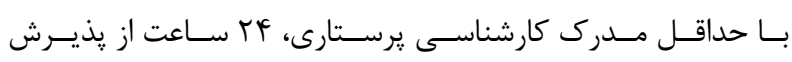

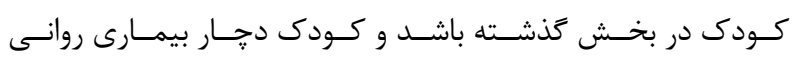

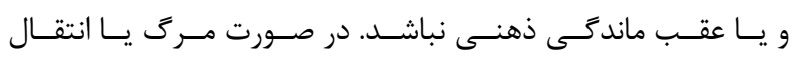

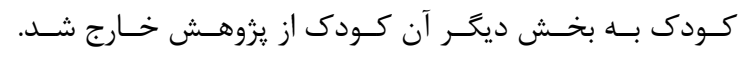

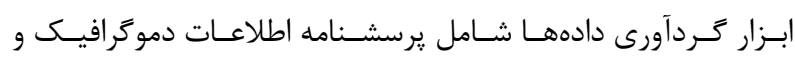

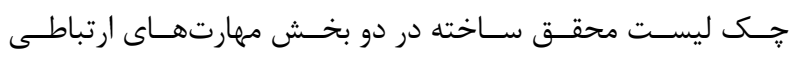

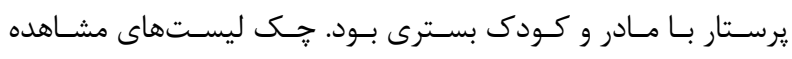

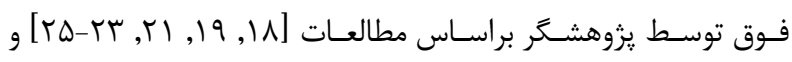




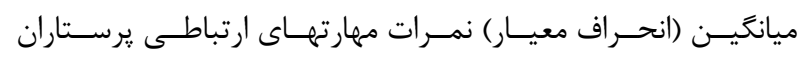

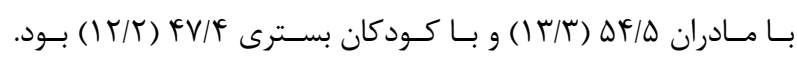

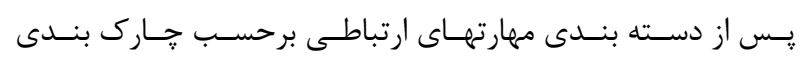

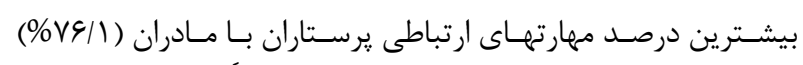

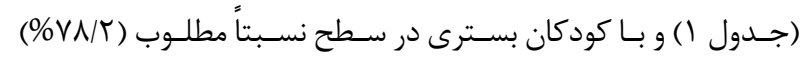

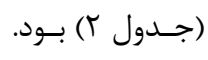

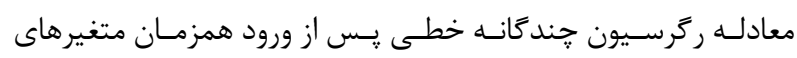

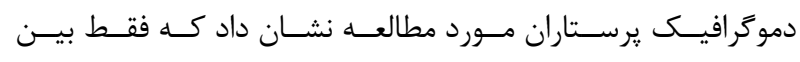

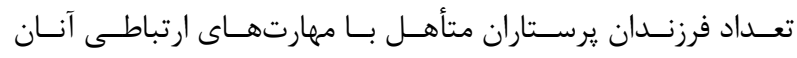

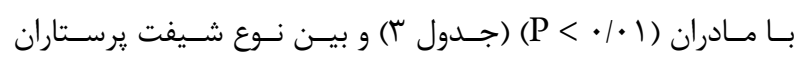

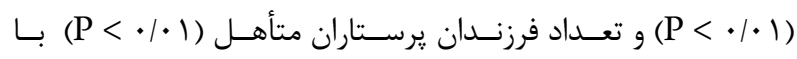

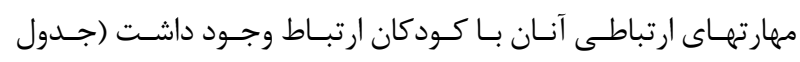

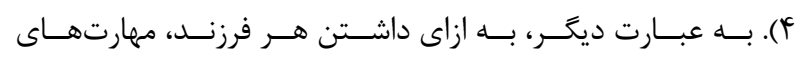

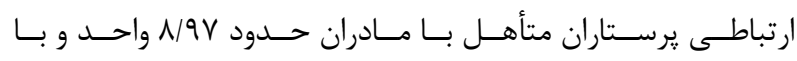

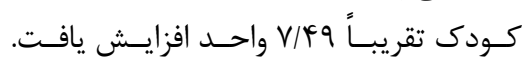

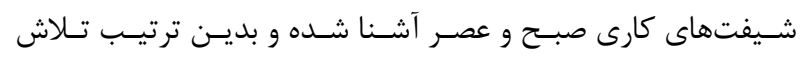

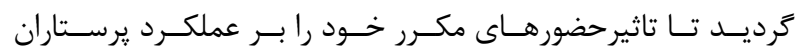
كاهـش دهــد.

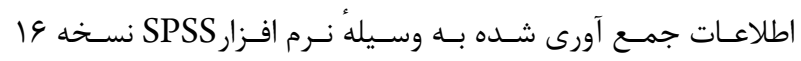

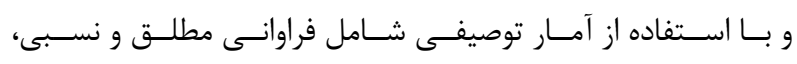

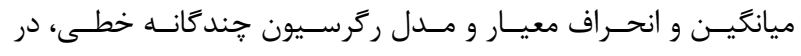

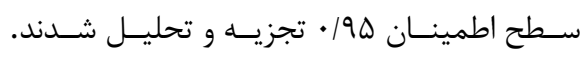

يافته ها

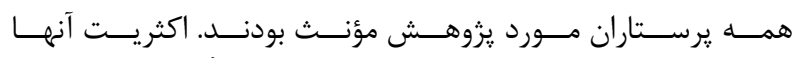

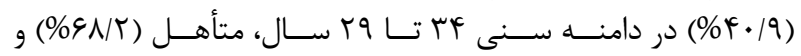

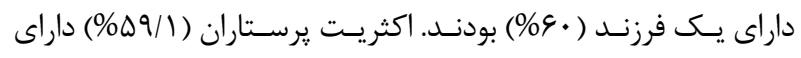

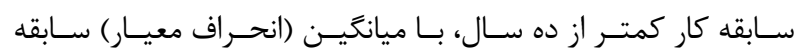

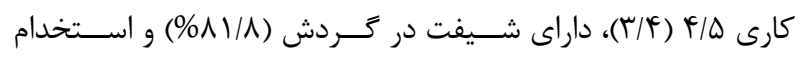

\begin{tabular}{|c|c|c|}
\hline \multicolumn{3}{|c|}{ جدول ا: توزيع فراوانى مهارتهاى ارتباطى يرستاران مورد مطالعه با مادران برحسب سطح آن } \\
\hline ميانگين \pm انحراف معيار & فراوانى (\%) & 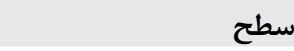 \\
\hline$r / 9 \pm r r / 4$ & rl $(9 / 4)$ & 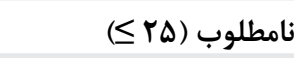 \\
\hline $\mid r / r \pm \Delta F / \Delta$ & TQI (VG/I) & نسبتاً مطلوب (r山 -VA) \\
\hline$\varphi \pm \Lambda r / \varphi$ & $\forall \wedge(\mid F / \Delta)$ & مطلوب ( ( \\
\hline$\Lambda \Lambda / 9 \pm \Delta \Delta / V$ & rr. $(1 \cdot \cdot)$ & 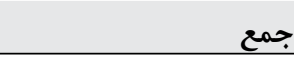 \\
\hline
\end{tabular}

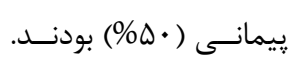

\begin{tabular}{|c|c|c|}
\hline \multicolumn{3}{|c|}{ جدول r: توزيع فراوانى مهارتهاى ارتباطى يرستاران مورد مطالعه با كودكان بسترى برحسب سطح آن } \\
\hline ميانگين 土 انحراف معيار & فراوانى (\%) & سطح \\
\hline$r / 9 \pm r / / 9$ & $4 \varphi(1 \% / 9)$ & 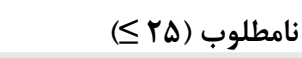 \\
\hline $\mid r / T \pm F V / F$ & $r \Delta \Lambda(V \Lambda / r)$ & 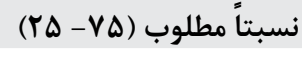 \\
\hline $9 / 1 \pm \lambda r / r$ & $r \varphi(V / 9)$ & مطلوب ( \\
\hline $\mid V / 9 \pm r q / 9$ & $r \mu \cdot(1 \cdot \cdot)$ & 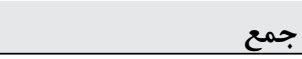 \\
\hline
\end{tabular}

\begin{tabular}{|c|c|c|c|c|c|}
\hline \multirow[b]{2}{*}{ P-Value } & \multicolumn{5}{|c|}{ جدول rا: آزمون ركر سيون حندَانه خطى تعيين ارتباط بين مشخصات دموكر افيك و مهار تهاى ارتباطى يرستاران با مادران } \\
\hline & مقاير & $\beta$ & انحر اف معيار & B & مشخصات دموكرافيك \\
\hline$<\cdot / \cdot 1$ & $\Delta / \Delta \mid$ & & $|r| \cdot r$ & $V 1 / 9 r$ & 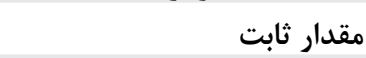 \\
\hline$\cdot \pi \& V$ & $-1 / 19$ & $\cdot \cdot / 1 r 1$ & $\cdot / 4 \mathrm{~V}$ & $-\bullet / \Delta \Delta$ & سن، سال \\
\hline.. .1 & $r / \mu_{1}$ & $\cdot / \mu \cdot 9$ & T/VT & N/9V & 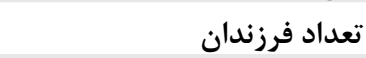 \\
\hline \multirow[t]{2}{*}{$\cdot \operatorname{lnIT}$} &.$/$ & $\cdot / \cdot r$. &.$/ 4 \Delta$ &.$/ 194$ & سابقه كارى، سال \\
\hline & & & & & نوع شيفت (مرجع = صبح) \\
\hline$\cdot 1 \cdot \Delta V$ & $-1 / 91$ &.$- / 19$. & r/Ar & $-9 / \pi r$ & مر كردش \\
\hline \multirow[t]{2}{*}{$\cdot / r \Delta V$} & $-1 / 1 F$ & $-\cdot / 1 \cdot \Delta$ & $\Delta / 99$ & $-9|1|$ & ثابت عصر \\
\hline & & & & & وضعيت استخدامى (مرجع = طر \\
\hline$\cdot / 49$ & .199 & $.1 .9 V$ & $\Delta / 9 \mathrm{~V}$ & $r / 91$ & رسمى \\
\hline \multirow[t]{2}{*}{.191} & $\cdot|4|$ & $.1 \cdot 4 \Delta$ & $\varphi / 19$ & $1 / 99$ & ي ي يمانى \\
\hline & & & & & وضعيت تأهل (مرجع = متأهل) \\
\hline . MFFF &.$/ 90$ & $\cdot \mid \cdot v 1$ & $r / \cdot V$ & r/q. & مجرد \\
\hline
\end{tabular}

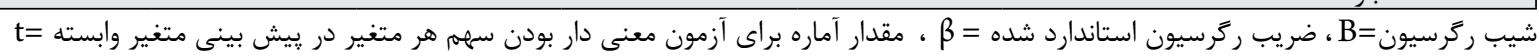




\begin{tabular}{|c|c|c|c|c|c|}
\hline \multirow[b]{2}{*}{ P-Value } & \multicolumn{5}{|c|}{ 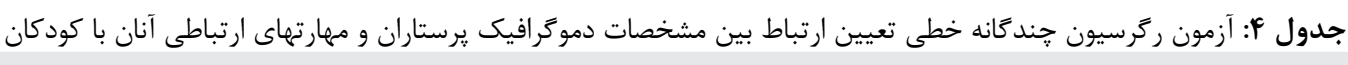 } \\
\hline & t & $\boldsymbol{\beta}$ & انحراف معيار & B & مشخصات دموگر افيك \\
\hline$>\cdot 1 \cdot \cdot 1$ & $9 \vee / 1 \wedge$ & & $\cdot / \mathrm{NT}$ & $\vee 1 / 09$ & مقدار ثابت \\
\hline$\cdot / \Delta V \Delta$ & $-\cdot \mid \Delta \& T$ & -.1 .91 & $\cdot / \cdot r \Delta$ & $-\cdot / 4 r q$ & سن، سال \\
\hline$* \cdot \cdot \cdot r$ & $r / \cdot 94$ & $\cdot / T V V$ & $\cdot 110$ & $V / 4 q$ & تعداد فرزندان \\
\hline \multirow[t]{2}{*}{.$/ 9 T V$} & $\cdot 1 \cdot 91$ & $\cdot / \cdot v$ & $\cdot / \cdot r f$ & $\cdot 1 \cdot r 4$ & سابقه كارى، سال \\
\hline & & & & & نوع شيفت (مرجع = ص \\
\hline$* \cdot 1 \cdot \cdot 1$ & $-\varphi / 4 \varphi$ & $-\cdot \mid$ \&MI & $\cdot / \pi q$ & $-19 / 4 \pi$ & در كردش \\
\hline \multirow[t]{2}{*}{$* \cdot 1 \cdot \cdot 1$} & $-\boldsymbol{F} / \Lambda$. &.$- / A T R$ & 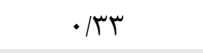 & $-r \Delta / \wedge q$ & ثابت عصر \\
\hline & & & & & وضعيت استخدامى (مرجع = \\
\hline$\cdot 10$ & $-\cdot 19 \Lambda$ &.$- / .94$ & $\cdot / \mu$ & $-r / 4 q$ & رسمى \\
\hline \multirow[t]{2}{*}{$\cdot 19$} &.$- \mid \Delta r$ & $-\cdot / \cdot \Delta \varphi$ &.$/ T T V$ & $-1 / 91$ & ي بيمانى \\
\hline & & & & & وضعيت تأهل (مرجع = متأهر \\
\hline$\cdot|r \Delta|$ & 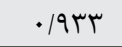 & $-.1 .9 V$ & $r / \Delta \Lambda$ & $-Y / \Delta V$ & مجرد \\
\hline
\end{tabular}

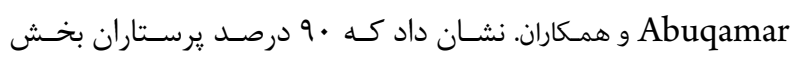

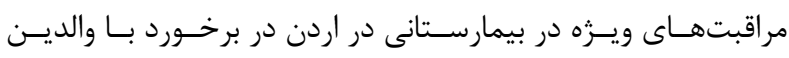

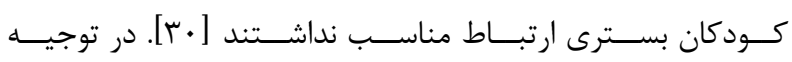

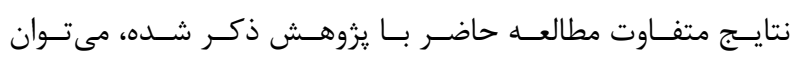

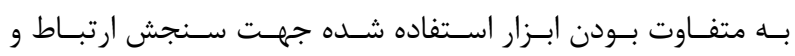

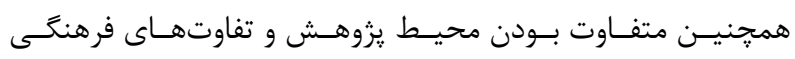

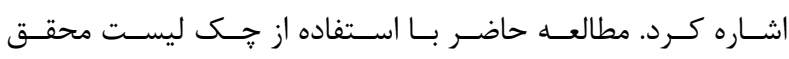

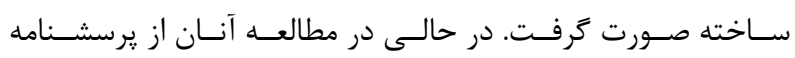

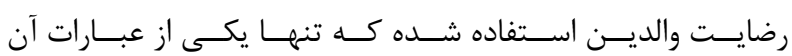

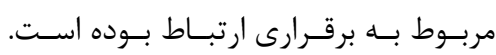

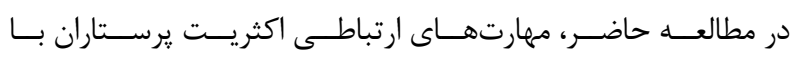

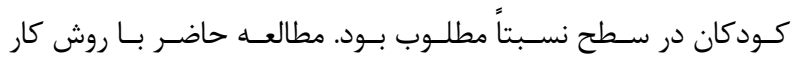

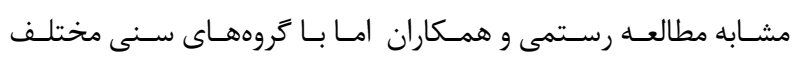

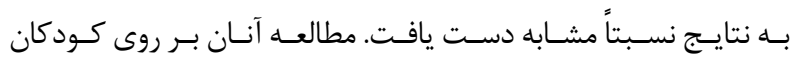

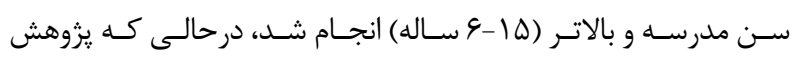

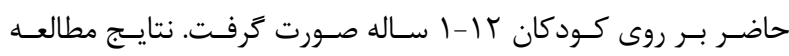

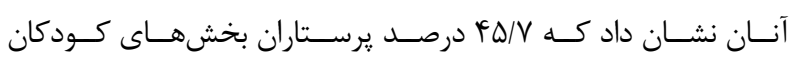

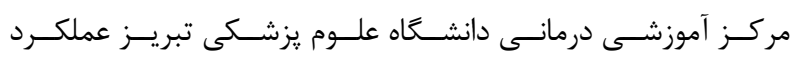

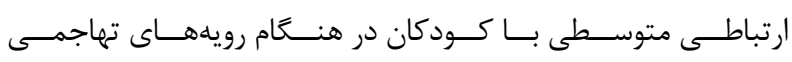

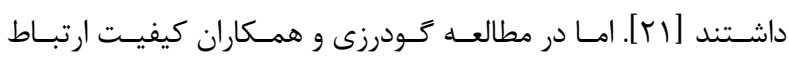

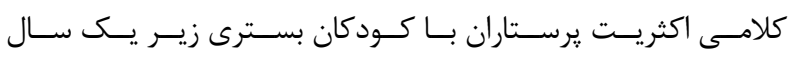

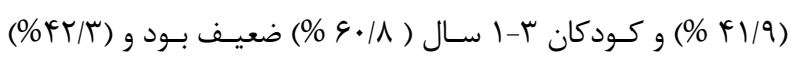

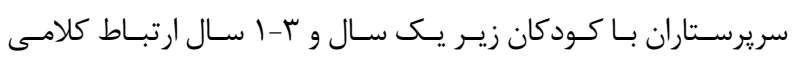

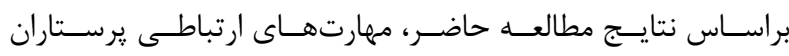

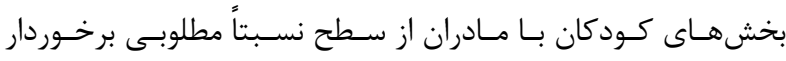

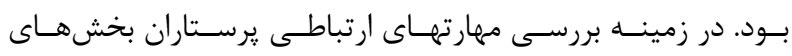

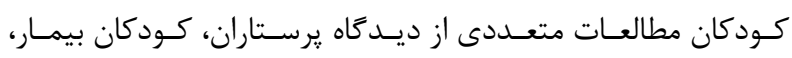

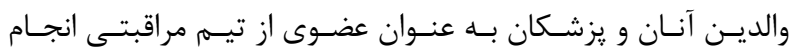

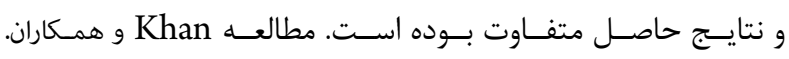

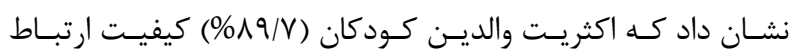

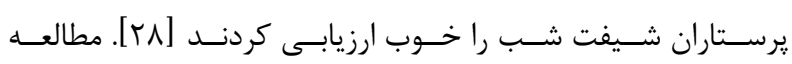

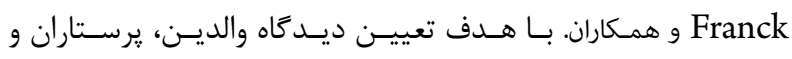

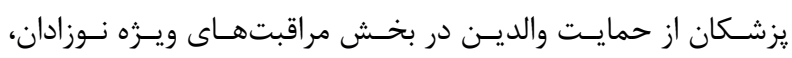

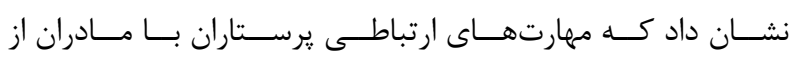

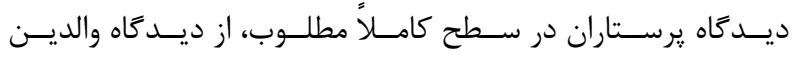

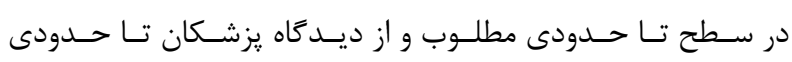

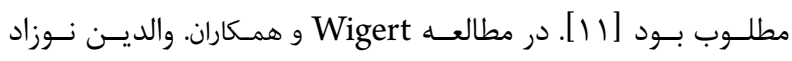

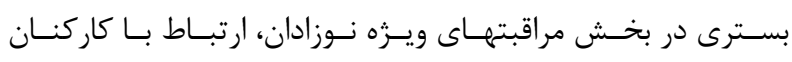

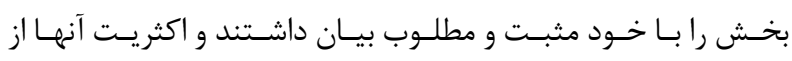

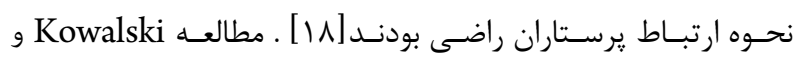

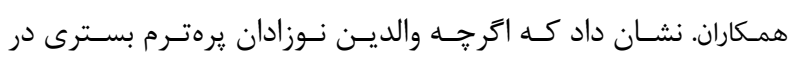

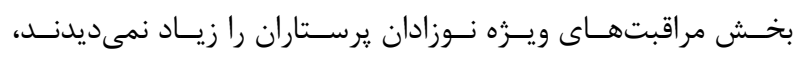

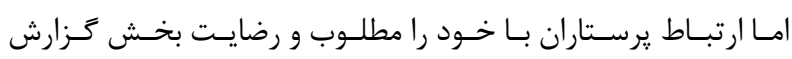

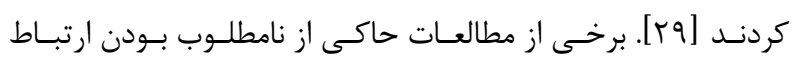

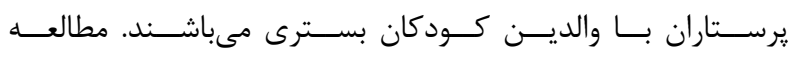




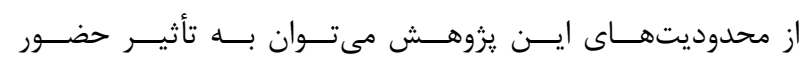

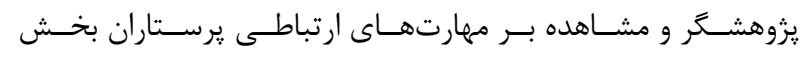

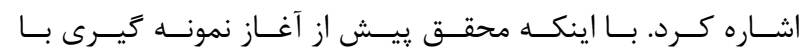

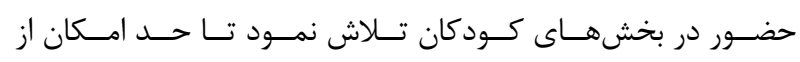

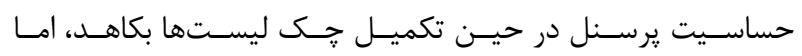

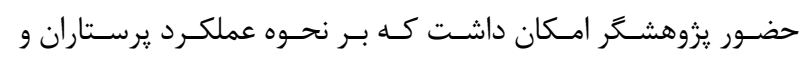

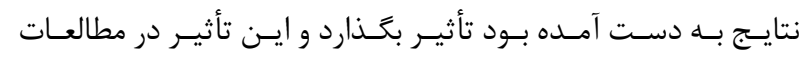

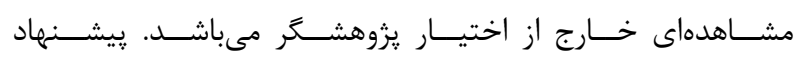

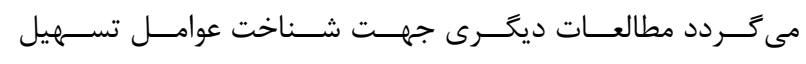

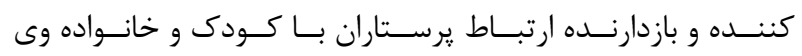

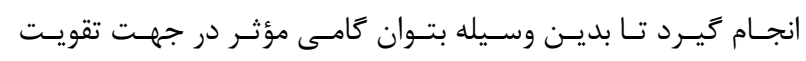

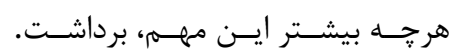

\section{نتيجه كيرى}

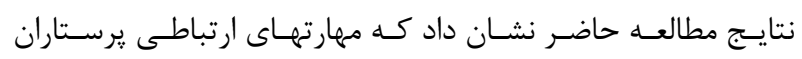

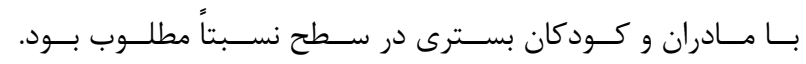

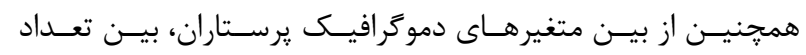

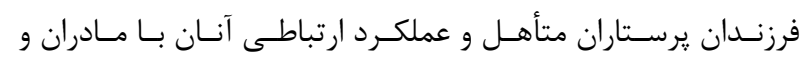

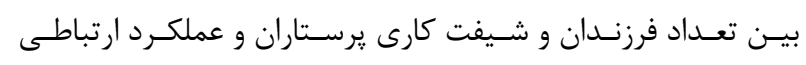

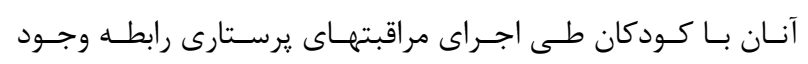

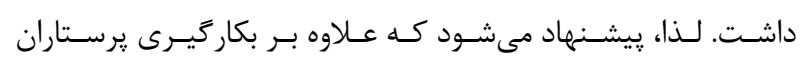

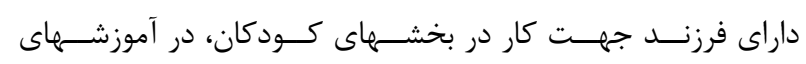

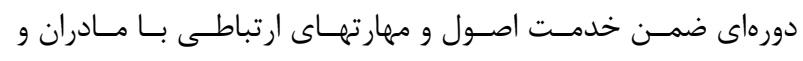

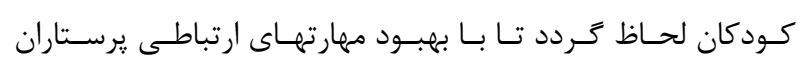

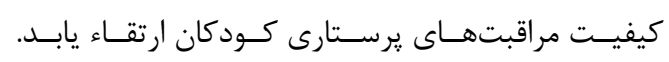

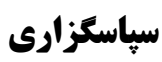

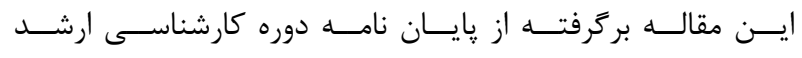

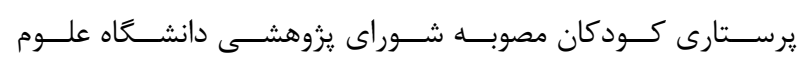

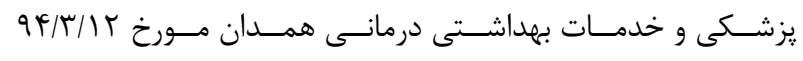

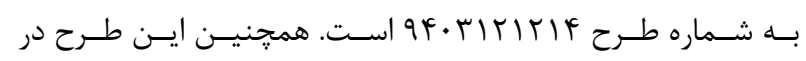

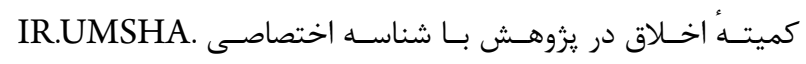

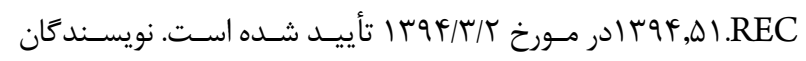

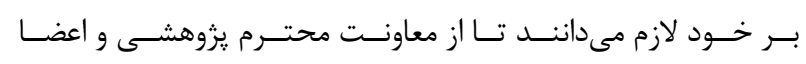

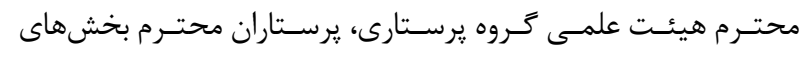

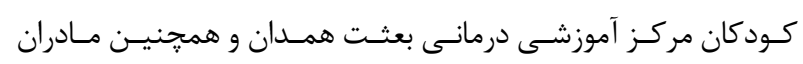

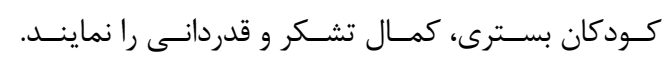

تضاد منافع

در اين مطالعه تضاد منافع وجود ندارد.
خوبسى داشـتند [9 9 ]. مطالـهـ Pelander و همـكاران. برخـلاف مطالعـه

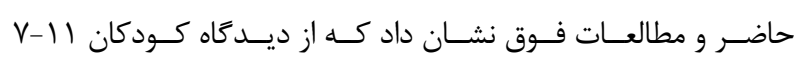

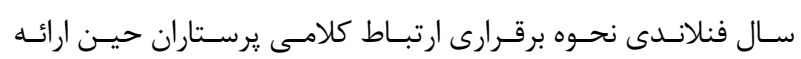

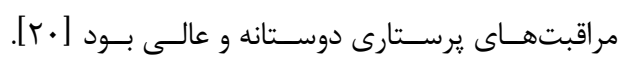

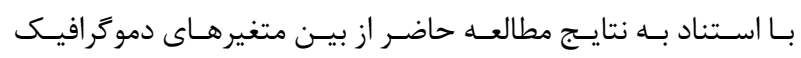

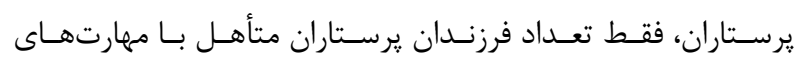

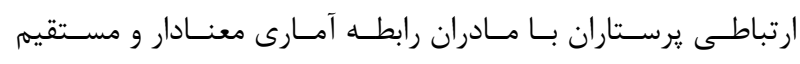

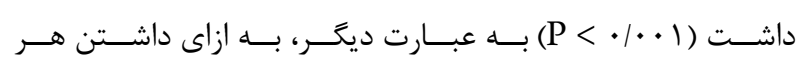

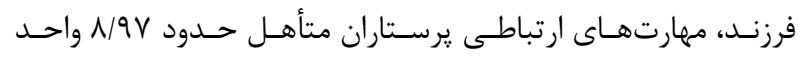

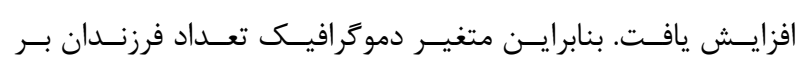

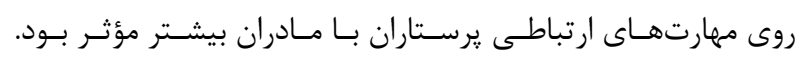

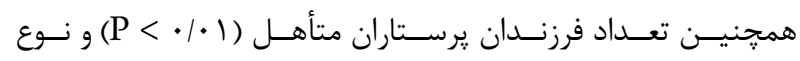

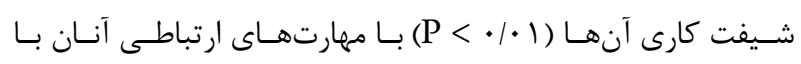

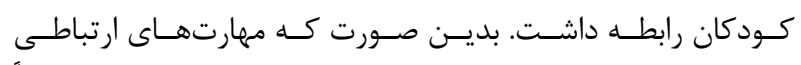

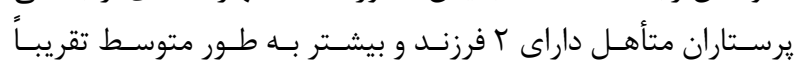

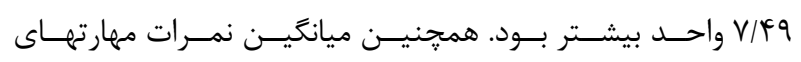

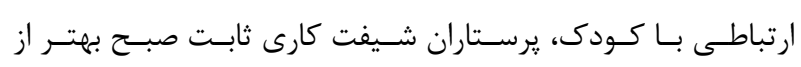

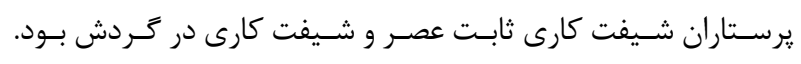

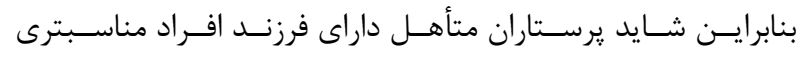

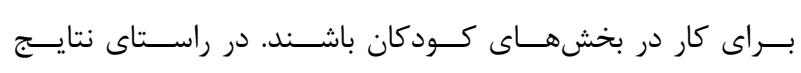

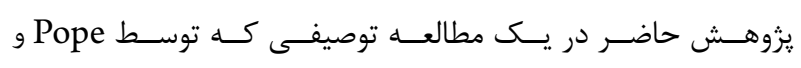

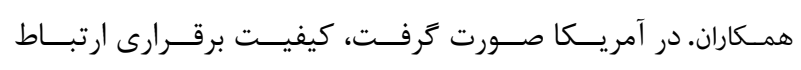

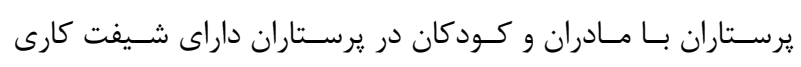

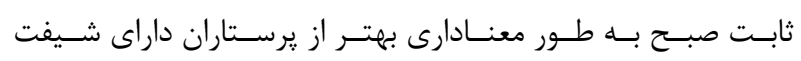

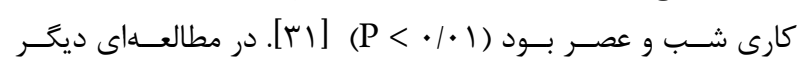

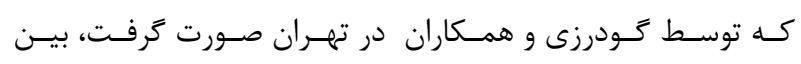

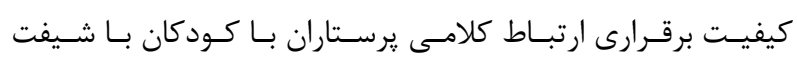

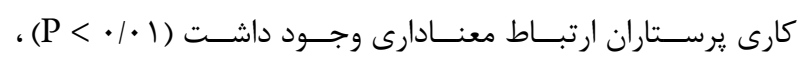

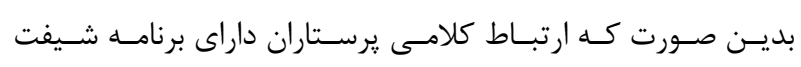

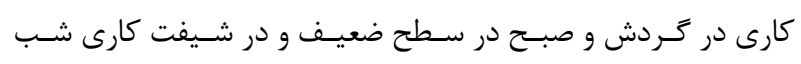

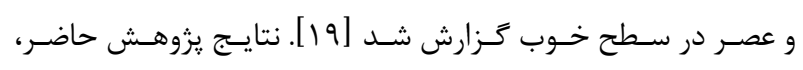

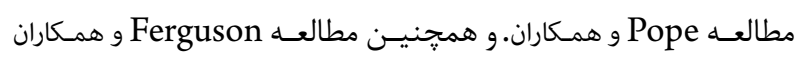

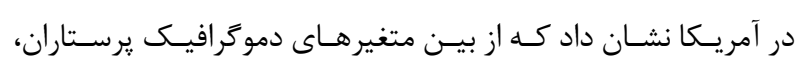

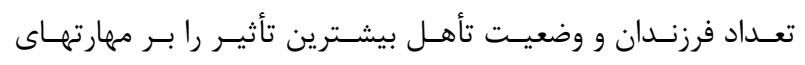

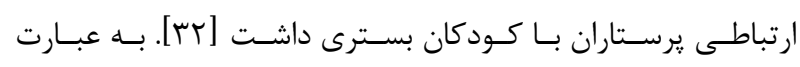

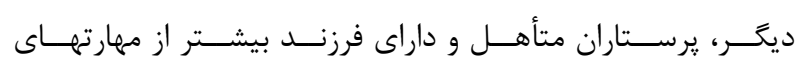

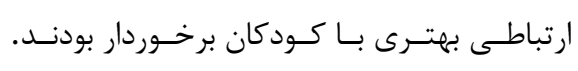

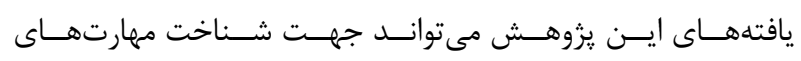

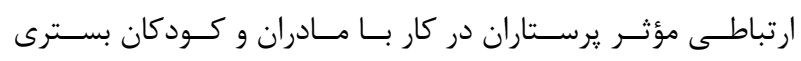

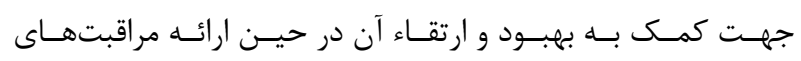

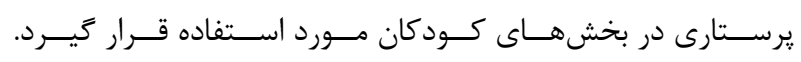




\section{REFERENCES}

1. Fisher MJ, Broome ME, Friesth BM, Magee T, Frankel RM. The effectiveness of a brief intervention for emotion-focused nurse-parent communication. Patient Educ Couns. 2014;96(1):72-8. DOI: 10.1016/j. pec.2014.04.004 PMID: 24797409

2. Mohammadi R, Seyedjavadi M, Mazaheri E. [Maternal Anxiety in Hospitalized Infants in Boali Training-Therapeutic Hospita in 2011]. Zanjan Univ Med Sci J. 2013;21(85):85-92.

3. Hockenberry M, Wong L, Wilson D. Wong's Nursing Care of Infants and Children. 9th ed. Canada: Elsevier Mosby; 2011.

4. Morsy AAK. Nursing support and stress among mothers of hospitalized children.J Am Sci. 2012;8(1s).

5. Pilliteri A. Maternal \& child health nursing: Care of the Childbearing \& Childrearing Family. 7th ed. Philadelphia: Lippincott Williams \& Wilkins; 2014

6. Kounenou K, Aikaterini K, Georgia K. Nurses' communication skills: Exploring their relationship with demographic variables and job satisfaction in a Greek sample. Procedia Soc Behav Sci. 2011;30:2230-4.

7. Mirhaghjou S, Nayebi N, Teymouri M, Kazemnejad L. [Communication skills and related factors within patient by nursing student]. Holist Nurs Midwife J. 2015;25(2):93-101.

8. Duzkaya DS, Uysal G, Akay H. Nursing perception of the children hospitalized in a university hospital. Procedia Soc Behav Sci. 2014;152:362-7.

9. Corsano P, Majorano M, Vignola V, Guidotti L, Izzi G. The waiting room as a relational space: young patients and their families' experience in a day hospital. Child Care Health Dev. 2015;41(6):1066-73. DOI: 10.1111/cch.12239 PMID: 25707424

10. Fisher M. A brief intervention to improve emotion-focused communication between newly licensed pediatric nurses and parents: Indiana University; 2012.

11. Alavi A, Bahrami M, Zargham-Boroujeni A, Yousefy A. Characteristics of caring self-efficacy in pediatric nurses: a qualitative study. J Spec Pediatr Nurs. 2015;20(3):157-64. DOI: 10.1111/jspn.12110 PMID: 25845908

12. Crawford R. Emotional communication between nurses and parents of a child in hospital. New Zealand: University of Technology Sydney; 2014.

13. Seyedamini B. [Fears, needs and nursing support of mothers during their child's hospitalization]. Iran J Nurs. 2011;24(27):57- 66

14. Sadeghi Shermeh M, Amiri H, Karimi Zarchi A, Bahari F, Binesh A. [Effectiveness of solution-focused communication training [SFCT] on nurses communication skills]. Iran J Military Med. 2013;14(4):269 -75.

15. Sepehri Nia M, Rassouli M, Alaee Karahroudi F, Zayeri F, Zagheri Tafreshi M. [Comparing perception of nurse-mother communication between nurses and mothers' hospitalized children]. QJ Nurs Manage. 2013;2(3):52-9.

16. Sousa P, Antunes A, Carvalho J, Casey A. Parental perspectives on negotiation of their child's care in hospital. Nurs Child Young People. 2013;25(2):24-8. PMID: 23586180

17. Long CO. Ten best practices to enhance culturally competent communication in palliative care. J Pediatr Hematol Oncol. 2011;33 Supp 2:S136-9. DOI: 10.1097/MPH.0b013e318230dfb4 PMID: 21952571 18. Wigert H, Dellenmark MB, Bry K. Strengths and weaknesses of par- ent-staff communication in the NICU: a survey assessment. BMC Pediatr. 2013;13:71. DOI: 10.1186/1471-2431-13-71 PMID: 23651578

19. Godarze Z, Darvesh A, Neli F. [Assess the quality verbal communication with children under three years of treatment in hospital]. Shahid Beheshti Nurs Midwife Fac. 2009;19(67):13-20.

20. Pelander T, Leino-Kilpi H, Katajisto J. The quality of paediatric nursing care: developing the Child Care Quality at Hospital instrument for children. J Adv Nurs. 2009;65(2):443-53. DOI: 10.1111/j.1365 2648.2008.04875.x PMID: 19016922

21. Rostami F, Mohamad por asel AFA. [Survey performance nurses pediatric educational hospitals of Tabriz with children during medical procedures]. Univ Med Sci Mazandaran. 2005;16(52):85-91.

22. Taylor C, Lillis C, Lemone P, Lynn P. Fundamental of Nursing the Art and Science of Nursing Care. 7th ed. Philadelphia: Lippincott Williams \& Wilkins 2011

23. Sabzevari S, Soltani Arabshahi K, Shekarabi R, Koohpayehzadeh J. [Nursing Students' Communication with Patients in Hospitals Affiliated to Kerman University of Medical Sciences]. Iran J Med Educ. 2006;6(1):43 - 8 .

24. Shin $H$, White-Traut R. Nurse-child interaction on an inpatient paediatric unit. J Adv Nurs. 2005;52(1):56-62. DOI: 10.1111/j.1365 2648.2005.03564.x PMID: 16149981

25. Schmidt C, Bernaix L, Koski A, Weese J, Chiappetta M, Sandrik K. Hospitalized children's perceptions of nurses and nurse behaviors. MCN Am J Matern Child Nurs. 2007;32(6):336-42; quiz 43-4. DOI 10.1097/01.NMC.0000298126.32161.4c PMID: 17968213

26. Lambert V, Long T, Kelleher D. Communication Skills for Children's Nurses. UK: Mc Graw-Hill Education; 2012.

27. Potter PA, Perry AG, Hall A, Stockert Patricia A. Fundamentals of Nursing. Philadelphia: Mosby Inc; 2013.

28. Khan A, Rogers JE, Melvin P, Furtak SL, Faboyede GM, Schuster MA, et al. Physician and Nurse Nighttime Communication and Parents' Hospital Experience. Pediatrics. 2015;136(5):e1249-58. DOI 10.1542/peds.2015-2391 PMID: 26504131

29. Kowalski WJ, Leef KH, Mackley A, Spear ML, Paul DA. Communicating with parents of premature infants: who is the informant? J Perinatol 2006;26(1):44-8. DOI: 10.1038/sj.jp.7211409 PMID: 16292336

30. Abuqamar M, Arabiat DH, Holmes S. Parents' Perceived Satisfaction of Care, Communication and Environment of the Pediatric Intensive Care Units at a Tertiary Children's Hospital.J Pediatr Nurs. 2016;31(3):e17784. DOI: $10.1016 /$ i.pedn.2015.12.009 PMID: 26803562

31. Pope CA, Escobar-Gomez M, Davis BH, Roberts JR, O’Brien ES Hinton E, et al. The challenge of tetradic relationships in medically interpreted pediatric primary care visits: A descriptive study of communication practices. Patient Educ Couns. 2016;99(4):542-8. DOI: 10.1016/i.pec.2015.10.032 PMID: 26796067

32. Hendricks-Ferguson VL, Sawin KJ, Montgomery K, Dupree C, Phillips-Salimi CR, Carr B, et al. Novice Nurses' Experiences With Palliative and End-of-Life Communication. J Pediatr Oncol Nurs. 2015;32(4):240-52. DOI: 10.1177/1043454214555196 PMID 25556105 


\title{
Nurses' Communication Skills With Mothers and Hospitalized Children During Nursing Care at the Children's Wards
}

\author{
Fatemeh Cheraghi ${ }^{1}$, Amenh Sanahmadi ${ }^{2}$, Ali Reza Soltanian ${ }^{3}$, \\ Amir Sadeghi ${ }^{4, *}$ \\ ${ }^{1}$ Associate Professor, Research Center for Chronic Disease (home care), School of \\ Nursing and Midwifery, Hamadan University of Medical Sciences, Hamadan, Iran \\ ${ }^{2}$ MSc. Student of Pediatric Nursing, School of Nursing and Midwifery, Hamadan \\ University of Medical Sciences, Hamadan, Iran
}

${ }^{3}$ Associate Professor, Research Center for Modeling of Noncommunicable Disease, Department of Biostatistics, School of Public Health, Hamadan University of Medical Sciences, Hamadan, Iran

${ }^{4}$ Assistant Professor, Research Center for Child \& Maternity Care, School of Nursing and Midwifery, Hamadan University of Medical Sciences, Hamadan, Iran

Corresponding author: Amir Sadeghi, Assistant Professor, Research Center for Child \& Maternity Care, School of Nursing and Midwifery, Hamadan University of Medical Sciences, Hamadan, Iran. Tel: +98-8138380535, E-mail: sadeghi@umsha.ac.ir

DOI: $10.20286 / \mathrm{nmj}-24037$

Received: 07.03.2016

Accepted: 23.04.2016

\section{Keywords:}

Communication

Nurses

Child, Hospitalized

Mothers

Pediatric Nursing

\section{How to Cite this Article:}

Cheraghi F, Sanahmadi A, Soltanian A, Sadeghi A. Nurses' Communication Skills With Mothers and Hospitalized Children During Nursing Care at the Children's Wards. Sci J Hamadan Nurs Midwifery Fac. 2016;24(3):193-200. DOI: $\quad 10.20286 / \mathrm{nmj}-24037$

(C) 2016 Scientific Journal of Hamadan Nursing \& Midwifery Faculty

\section{Abstract}

Introduction: Nurses relationship with children and their family can be effective in the stress of hospitalized children. The aim of this study was to determine the communication skills of nurses when dealing with children and their mothers hospitalized at pediatric wards of Besat Educational and Treatment Center in Hamadan.

Methods: In this descriptive-analytical study, using the census method, 22 pediatric nurses were selected. After that, using simple random sampling, communication skills of nurses when dealing with hospitalized children and their mothers were assessed for 330 cases during nursing care. In order to collect data, researcher-a made Communication Skill Checklist with mothers and children were employed. Using descriptive statistics and multiple linear regression model, data were analyzed by SPSS software version 16.

Results: Seventy-six percent of nurses' communication skills with mothers (with mean value of 54.5 and standard deviation value of 13.3) and with children (with mean value of 47.4 and standard deviation value of 12.2 ) were relatively desirable. Multiple linear regression equation showed that among nurses' demographic characteristics, only the number of children of married nurses had a statistical relationship with their communication skills with mothers $(\mathrm{P}<0.01)$. Also type of work shift $(\mathrm{P}<0.01)$ and number of children of married nurses $(\mathrm{P}<0.01)$ had a statistical relationship with their communication skills with children.

Conclusions: Although nurses' communication skills with mothers and hospitalized children were relatively high but improvement of the quality of nursing care needs to be promoted. It is recommended for in-service training courses to be held in this field. 